\title{
LA IDENTIDAD ÉTNICA DE ESTUDIANTES ARAWAK EN LA UNIVERSIDAD CATÓLICA SEDES SAPIENTIAE (ATALAYA)
}

\author{
Liliana Fernández Fabián* \\ Instituto de Investigaciones y Desarrollo Andino \\ imatlife@yahoo.com
}

Fecha de recepción: agosto de 2016 Fecha de aceptación: diciembre de 2016

RESUMEN: Este documento es el resultado de una etnografía de aula aplicada a los alumnos ingresantes al primer ciclo del Programa Magisterial de Formación de Docentes en Educación Básica Intercultural Bilingüe de la Universidad Católica Sedes Sapientiae, sede Atalaya, durante el Año Académico 2015. El objetivo de este estudio es mostrar la influencia de la Educación Intercultural Bilingüe a nivel superior en la construcción de la identidad de jóvenes de pueblos Arawak. Este programa ofrece un contexto social diverso en el cual los estudiantes que provienen de diversos

* Liliana Fernández Fabián es licenciada en Lingüística y candidata a obtener el grado de magíster en Antropología en la Universidad Nacional Mayor de San Marcos con su tesis Construcción identitaria de los jóvenes ashéninkas del Gran Pajonal en el siglo XXI. Ha sido colaboradora del Centro de Investigación de Lingüística Aplicada (CILA) y del Instituto de Investigaciones Lingüísticas (INVEL). Ha trabajado en varias universidades nacionales y en la Universidad Católica Sedes Sapientiae, sede Atalaya. Ha escrito numerosos artículos sobre la gramática de la lengua ashéninka y ha participado en eventos académicos nacionales e internacionales como panelista. Actualmente es investigadora en el Instituto de Investigaciones y Desarrollo Andino. 
pueblos originarios conviven y construyen su identidad étnica sobre la base de semejanzas y diferencias que encuentran entre ellos. En este trabajo se ofrecerán datos etnolingüísticos de los pueblos Arawak, además de describir los cursos de lengua indígena. La conclusión es que la educación que reciben en la universidad favorece la producción de identidad al fortalecer las bases culturales propias.

Palabras Clave: Arawak, cultura, identidad étnica, indígena, revitalización lingüística

\section{EDUCATION AND IDENTITY IN UNIVERSIDAD CATÓLICA SEDES SAPIENTIAE (ATALAYA)}

AвSTRACT: This paper is the result of an ethnographic study applied to university students of the first year in the Universidad Católica Sedes Sapientiae's Programa Magisterial de Formación de Docentes en Educación Básica Intercultural Bilingüe in Atalaya, during the 2015 academic year. The aim of this study is to show the influence of Intercultural Bilingual Education at university in the building identity of Arawak young people. This program offers a diverse social context in which students from various indigenous populations live and build their identity on the basis of similarities and differences among them. In this paper, data about Arawak populations is provided as well as describing the indigenous language courses. The conclusion is that the education received at the university favors the production of identity by strengthening their own cultural bases. KEYwords: Arawak, culture, ethnic identity, indigenous populations, linguistic revitalization 


\section{Introducción}

T a Universidad Católica Sedes Sapientiae tiene una sede en la ciudad de Atalaya (Ucayali) donde la mayoría de alumnos matriculados son de origen indígena, ya que proceden de diversos pueblos originarios de la Amazonía, principalmente de las familias etnolingüísticas Pano y Arawak de la Selva Central del Perú. Al llegar a este centro de estudios, muchos de los alumnos se enfrentan por primera vez a la diversidad multicultural y plurilingüe de nuestro país. Quedan sorprendidos de ver que existen otros pueblos indígenas, que a sus compañeros no les da vergüenza vestir su indumentaria tradicional ni comunicarse en su lengua materna, una lengua indígena.

No obstante, también ocurre que algunos jóvenes, si bien tienen ascendencia indígena, se han criado en ciudades por motivos laborales de sus progenitores o porque alguno de ellos es mestizo. Esto ha provocado un desarraigo cultural que se manifiesta en que no hablen la lengua de su pueblo como resultado de la propia determinación o porque sus padres les negaron ese derecho. Algunos jóvenes, incluso, desconocen la historia y los patrones culturales con los cuales podrían decir que pertenecen a uno u otro pueblo indígena.

Los ingresantes a la carrera de Educación Intercultural Bilingüe en el año 2015 fueron matriculados en cursos de Lengua indígena según sus pueblos originarios: shipibo, asháninka, ashéninka, yine, yánesha, matsigenka y nomatsigenga, dado que se contaba con un profesor para cada uno de estos grupos. Asimismo, en las tardes, una vez por semana, asistían al taller de Artesanía indígena. Los demás cursos los llevaban todos juntos de acuerdo al Plan curricular de sus respectivas carreras. 
Durante el primer Semestre Académico, estos alumnos compartieron las clases de Lengua con alumnos de las carreras de Administración e Ingeniería Agraria. Estos alumnos bien pertenecían a algún pueblo indígena mencionado o bien se inscribían en alguno de los cursos por afinidad social. Entonces, en este contexto, los jóvenes Arawak construían su identidad étnica, basándose en las diferencias y semejanzas que encontraban entre sus pares y en los diálogos interculturales con sus compañeros y docentes.

En la Universidad, la interculturalidad rige como principio y norma social en la vida de los alumnos que viven en el albergue y, en las clases, como eje que guía la conducta entre indígenas de distintos pueblos originarios del país y mestizos. De ese modo, los alumnos se sienten libres para manifestar abiertamente sus creencias y prácticas culturales y aprender de los demás. Esto no impide cuestionamientos y rechazos hacia ciertas conductas, pero el clima de respeto y tolerancia es el que siempre prevalece.

El objetivo principal de este trabajo, entonces, es describir el proceso de construcción de la identidad étnica de los jóvenes procedentes de pueblos indígenas Arawak mediante la influencia que ejerce la universidad en su formación académica. La información ha sido ordenada de la siguiente forma: (a) la presente introducción, (b) pueblos Arawak, (c) características socio-históricas de los alumnos, (d) proceso de construcción de la identidad étnica, (e) conclusión y (f) referencias.

Los resultados de este estudio se sustentan en la aplicación de una metodología basada principalmente en la observación participante y conversaciones con alumnos y docentes. Se ha considerado que la observación participante durante todo el año académico 2015 ha sido una técnica cualitativa importante para socializar con los alumnos y conocer su aproximación a la lengua y la cultura originarias de cada uno. 


\section{Pueblos Arawak}

Los pueblos indígenas Arawak en Perú son los siguientes: asháninka, ashéninka, kakinte, chamicuro, iñapari, matsigenka, nanti, nomatsigenga, resígaro, yánesha y yine. Además, actualmente existen en el Perú diversos pueblos indígenas en aislamiento voluntario y contacto inicial, cuyos idiomas han sido clasificados al interior de dos familias lingüísticas Arawak y Pano. Los pueblos Arawak que están en esta condición son los mashco piro, ubicados en la cuenca del río Purús (Ucayali), cuenca de los ríos Las Piedras y Manu (Madre de Dios); los matsigenkas han sido vistos en Bajo Urubamba (Cusco), río Manu y el alto Madre de Dios (Madre de Dios); los nanti, en los ríos Camisea, Timpía y Ticumpinía, entre las cuencas del Urubamba y Manu (Cusco y Madre de Dios); los caquinte en la cordillera de Vilcabamba (entre Junín y Cusco) (Ministerio de Educación, 2013). A continuación, la Tabla 1 mostrará la población reconocida como Arawak en el Perú se distribuye de la siguiente manera: 
Tabla 1

Ubicación Geográfica y Demografía

\begin{tabular}{llr}
\hline \multicolumn{1}{c}{ Pueblo } & \multicolumn{1}{c}{ Ubicación } & Población \\
\hline \multirow{2}{*}{ Asháninka } & Junín, Pasco, Ucayali, Apurímac, Ayacucho, & 88,703 \\
& Huánuco, Cusco, Lima, Loreto & \\
Ashéninka & Pasco, Junín, Ucayali & 8,774 \\
Matsigenka & Cusco, Madre de Dios, Ayacucho, Lima & 11,275 \\
Nomatsigenga & Junín & 8,016 \\
Yánesha & Pasco, Junín, Huánuco & 7,523 \\
Yine & Ucayali, Cusco, Madre de Dios, Loreto & 3,261 \\
Kakinte & Junín, Cusco & 439 \\
Nanti & Cusco & 250 \\
Chamicuro & Loreto & 63 \\
Resígaro & Loreto & 37 \\
Iñapari & Madre de Dios & 4 \\
\hline
\end{tabular}

Nota. Adaptado de "II Censo de Comunidades Indígenas de la Amazonía Peruana 2007. Resultados definitivos," por Instituto Nacional de Estadística e Informática, 2007. "Documento nacional de lenguas originarias del Perú," por Ministerio de Educación, 2013.

\section{Características Sociohistóricas de los Alumnos}

La ciudad de Atalaya se ubica en la parte suroeste del departamento de Ucayali y limita con los departamentos de Junín y Pasco. Está rodeada por asentamientos de pueblos indígenas de la Selva Central del país. Las distancias geográficas son cortas, pero algunas vías que unen a estos pueblos son inhóspitas, lo cual dificulta el acceso a la ciudad. Aun así, cada año aumenta el número de alumnos que eligen la Universidad Católica Sedes Sapientiae como alternativa para su desarrollo profesional. En lo que continúa, se describirá las características sociohistóricas de los alumnos Arawak según los pueblos originarios a los cuales pertenecen. 


\subsection{Pueblo asháninka}

Los asháninkas son el pueblo indígena amazónico más numeroso del Perú y abarcan una gran y variada extensión geográfica que ha influido en sus modos de vida, además de algunas manifestaciones culturales. El pueblo asháninka habita en los departamentos de Junín, Ucayali, Huánuco, Cusco, Pasco y Ayacucho. Así, se distinguen entre ellos principalmente por las cuencas que se encuentran próximas a sus comunidades: los asháninkas de los valles del río Pichis y Perené; los asháninkas de los valles de los ríos Apurímac, Tambo y Ene; o los asháninkas de Ucayali, los cuales se diferencian principalmente por una forma particular del habla.

Aunque la lengua asháninka está considerada, dentro del parámetro de vitalidad de la lengua, como vital porque es hablada por todas las generaciones asháninka, no todos los alumnos que ingresan a la carrera de Educación Básica Intercultural Bilingüe en la UCSS tienen un mismo nivel de dominio de la lengua. Su competencia varía tanto a nivel oral como escrito, lo cual es un indicador de que las comunidades asháninkas viven distintos procesos de cambios socioculturales que afectan en cierta medida el uso de la lengua.

La mayoría de alumnos asháninkas son originarios de comunidades nativas del río Tambo, del río Ucayali y del Alto Perené. Al menos un alumno por ciclo tiene como lengua materna el castellano. Empero, han sido aceptados debido a su ascendencia asháninka y se encuentran aprendiendo la lengua como futuros docentes bilingües.

La cultura asháninka se ha visto expuesta a la influencia mestiza debido al contacto con occidente desde el periodo de la colonización (siglo XVII) que trajo consigo esclavitud, epidemias y muerte de miles de asháninkas. Durante los siglos XIX y XX, poblaciones de origen europeo y 
andino lograron obtener parte de sus tierras, lo cual ocasionó el desplazamiento de muchos hacia las cuencas del río Ene y del río Tambo (Asociación Interétnica de Desarrollo de la Selva Peruana et al., 2000). La amenaza de perder el territorio, hizo que los asháninkas conformaran, en las décadas de 1970 y 1980, las primeras organizaciones indígenas para defenderse y luchar por sus derechos.

Asimismo, los asháninkas fueron uno de los pueblos indígenas más afectados por la violencia política. La Comisión de la Verdad y Reconciliación calcula que en las últimas dos décadas del siglo XX, unos 10,000 asháninkas fueron desplazados forzosamente en los valles del río Ene, Tambo y Perené; 6,000 fueron muertos y 5,000 de ellos fueron capturados por el grupo terrorista Sendero Luminoso. Asimismo, se estima que entre treinta y cuarenta comunidades asháninka habrían desaparecido durante esta época (Comisión de la Verdad y Reconciliación, 2003). Por su parte, los alumnos de la UCSS han vivido parte de esta violencia: algunos cuentan que han perdido a sus padres y hermanos en emboscadas, han vivido en el monte huyendo de los terroristas o han terminado en misiones alejados de sus comunidades.

En la actualidad, la mayoría de asháninkas ya no viven en asentamientos dispersos: ahora construyen sus casas alrededor de los servicios de salud y educación estatales, aunque las chacras se ubican al interior de los bosques, quebradas y ríos que hay en sus comunidades. Pocos mantienen una vida tradicional. Las nuevas generaciones participan activamente del desarrollo económico de sus comunidades, las cuales esperan ver convertidas en centros de desarrollo político y social.

Entre las prácticas económicas actuales están el comercio de madera y la producción de café y cacao. Sin embargo, las actividades tradicionales como la cacería y recolección aún se practican. La yuca sigue siendo el producto principal en la alimentación de este pueblo, pero ahora la dieta contiene otro 
tipo de carnes como las gallinas que crían o los pollos que compran en los mercados. Respecto a la vestimenta, las cushmas tradicionales han pasado a ocupar un lugar de representación simbólica de su etnicidad y visten, en cambio, ropas de origen occidental diariamente.

A pesar de los cambios socioculturales que han experimentado los asháninkas, muchas creencias ancestrales se mantienen incólumes. Entre ellas se aprecian la división de mundos, la vida en estos, y la existencia de seres sobrenaturales que cohabitan con los humanos la tierra. La relación enfermedad-salud encuentra sustento en la naturaleza y en estas creencias, de ahí que el conocimiento sobre los beneficios de algunas plantas se mantenga hasta la actualidad.

Los alumnos asháninkas que ingresan a la UCSS tienen diferentes niveles de conocimiento sociocultural. Algunos llegan a la universidad a reaprender su historia. Empero, los hechos de terror sufridos por sus familias y ellos mismos permanecen vigentes en sus memorias.

\subsection{Pueblo ashéninka}

Se autoidentifican como ashéninkas quienes habitan los territorios del Apurucayali, el Gran Pajonal, el Pichis, el sur de Ucayali y el territorio de Yurúa, en los departamentos de Ucayali y Pasco. Han sido relacionados con el pueblo asháninka debido a que tienen una historia común y a que tradicionalmente se han ubicado en la misma zona geográfica: la Selva Central del Perú. Además, ambos pueblos comparten una lengua que, aunque con distintas variantes, es considerada como una de las lenguas dentro de la familia lingüística Arawak.

Sin embargo, desde el año 2009, en la UCSS, se dictan clases de lengua ashéninka. Para ello, la responsable del dictado es la profesora 


\section{LILIANA FERNÁNDEZ FABIÁN}

Luzmila Casique Coronado, originaria de la comunidad nativa de Chicosa en Ucayali. Con esto se crea un espacio para la construcción de la identidad étnica de los alumnos quienes se distinguen de sus pares asháninkas principalmente por su indumentaria, costumbres y forma de hablar.

El Gran Pajonal constituye, quizás, la zona ashéninka más tradicional debido a que el proceso de recolonización se dio recién en 1935. Cabe agregar que este evento se dio casi doscientos años después de la rebelión de Juan Santos Atahualpa. Según Hvalkof y Veber (2005), el más grande logro de este levantamiento fue la expulsión de los misioneros y la recuperación del control sobre sus territorios.

No obstante, la historia de colonización es casi la misma que la que ocurrió con los asháninkas: epidemias, esclavitud y muerte. En la década de 1980, los grupos armados subversivos como el Movimiento Revolucionario Túpac Amaru (MRTA) y Sendero Luminoso comienzan a realizar acciones en territorios de los pueblos asháninka y ashéninka. En 1988, esta zona fue declarada en "estado de emergencia". Frente a la incursión terrorista, los ashéninkas conformaron el "Ejército Ashéninka" a fines de la década de 1980, logrando detener el ingreso de Sendero Luminoso a la zona del Gran Pajonal (Comisión de la Verdad y Reconciliación, 2003; Espinosa, 1993; Hvalkof \& Veber, 2005).

Esta acción y sus prácticas culturales ancestrales como la poliginia, el cultivo de ibenkis, plantas mágico-religiosas, el uso de la vestimenta tradicional y la pintura facial han hecho que los ashéninkas sean vistos por otros como un pueblo guerrero, tradicional, conocedor de los secretos del monte. Lo cierto es que las condiciones de acceso a este territorio siempre fueron difíciles, lo cual promovió su distanciamiento del desarrollo local en las ciudades cercanas. Así también, propició la continuidad de actividades económicas básicas como la caza, pesca, agricultura de subsistencia y recolección (Hvalkof \& Veber, 2005). 
En la actualidad, los ashéninkas que ingresan a estudiar a la UCSS son minoría. A la convocatoria anual responden jóvenes del río Ucayali y Gran Pajonal; no obstante, son pocos los interesados. Las razones se deben a un desinterés por la profesionalización debido al bajo nivel en la calidad educativa que existe en Ucayali. Incluso no todos acceden a la educación secundaria, ya que hay pocos colegios en la región. Por ejemplo, en la zona del Gran Pajonal, apenas hay tres colegios secundarios y de estos solo ingresan anualmente el $50 \%$ de alumnos que terminan la educación primaria. Los demás jóvenes optan por formar familias y trabajar su chacra.

Aunque en la UCSS los ashéninkas encuentran un espacio donde se reconoce su identidad, muy pocos han logrado concluir sus estudios. La mayoría ha dejado la universidad a mitad de la carrera. En el 2015, no egresó ningún alumno ashéninka y, en el 2016, solo egresará uno como docente bilingüe.

\subsection{Pueblo nomatsigenga}

El pueblo nomatsigenga está vinculado culturalmente con el pueblo Asháninka. Muchos nomatsigengas son trilingües (nomatsigenga, asháninka, castellano) debido a la cercanía de los territorios o por los matrimonios mixtos. Viven en la provincia de Satipo entre los ríos Ene, Perené y Anapati, en una zona donde el conflicto entre narcotraficantes, senderistas y las fuerzas armadas casi extermina por completo a la población en la década de los noventa (Comisión de la Verdad y Reconciliación, 2003). Además, las comunidades se encuentran próximas a las ciudades y la economía está influenciada por el narcotráfico (Mayor \& Bodmer, 2009).

La vulnerabilidad de este pueblo siempre ha estado marcada por el contacto con foráneos. Los misioneros, por ejemplo, tuvieron hasta 
38 puestos de evangelización hacia el año 1675 (Asociación Interétnica de Desarrollo de la Selva Peruana et al., 2000). En la segunda mitad del siglo XIX, el desarrollo agrícola y ganadero, así como el surgimiento de haciendas de café, caña de azúcar y frutales, propiciaron una masiva corriente colonizadora en el territorio nomatsigenga (Barclay, 2006). En la década de 1980, el Movimiento Revolucionario Túpac Amaru (MRTA) y Sendero Luminoso (SL) ocuparon zonas donde vivían los nomatsigengas y desplegaron numerosas acciones subversivas expandiéndose con fuerza por la región, llegando a dominar prácticamente toda la provincia de Satipo y obteniendo el control de los valles de los ríos Perené, Ene y Tambo (Comisión de la Verdad y Reconciliación, 2003; Espinosa, 1993).

En las últimas décadas, el pueblo nomatsigenga se ha integrado a la economía nacional con el comercio de café y cacao; los alumnos manifiestan que sus padres se dedican también a la crianza de aves de corral para la venta. Generalmente son los abuelos quienes mantienen sus prácticas culturales tradicionales: las mujeres nomatsigengas, por ejemplo, preparan el masato, elaboran atuendos y artículos decorativos en base a cortezas de árboles, semillas, dientes de animales y tintes naturales como el achiote.

Los jóvenes nomatsigengas tienen muchas posibilidades de acceder a la educación superior en las ciudades de Satipo y La Merced, por ejemplo. En la UCSS, en la primera promoción de estudiantes de Educación, hubo un alumno nomatsigenga llamado Hermías Delgado Inga. Al año siguiente de su ingreso, le siguieron su hermano menor junto a otro joven nomatsigenga. Estos alumnos y otros que ingresarían en los años consecutivos estudiaban asháninka. El año 2015, siete alumnos nomatsigengas ingresaron, por lo que se contrató a Hermías Delgado, ya egresado, para ser el profesor a cargo de dictar la cátedra nomatsigenga: el curso de Lengua y el curso de Artesanía. 


\subsection{Pueblo matsigenka}

Este pueblo se encuentra principalmente en los departamentos de Cusco y Madre de Dios. En este caso, quienes estudian en la UCSS proceden de Cusco. Ellos, desde hace cuatro años, son favorecidos con becas de estudios de la empresa Pluspetrol que extrae el gas de Camisea.

Las evidencias históricas de la presencia de este pueblo se remontan al periodo prehispánico. La inclusión de palabras de origen quechua en la lengua matsigenka, numerosos cuentos y mitos sobre el Inca y la tradición de tejer túnicas de algodón como las cushmas, serían las evidencias de la antigua relación comercial entre poblaciones andinas y este pueblo. Se cree que los matsigenkas proveían coca, tabaco, pieles y plumas a las poblaciones andinas, en tanto ellos recibían a cambio herramientas de bronce, sal y textiles (Shepard \& Izquierdo, 2003; Rosengren, 2004).

Entre los años 1799 y 1900, los misioneros franciscanos tuvieron a su cargo la evangelización de la zona del Urubamba con lo que se estableció cierto contacto con los matsigenkas. Los misioneros demostraron poco interés por la evangelización de este pueblo debido a su gran dispersión y a la ausencia de grandes aldeas (Rosengren, 2004). Sin embargo, cuando esta situación cambió y se inició la colonización con fuerza, se dio una alta mortalidad de la población indígena, principalmente en la época del boom del caucho. Recién a principios del siglo XX, con el establecimiento de misiones dominicas en Chirumbia, se puede considerar que empezó la evangelización de los matsigenkas, proceso que se desarrolló con mayor magnitud con la llegada del Instituto Lingüístico de Verano (ILV) en la década de 1950 (Instituto Lingüístico de Verano, 2006; Rosengren, 2004).

Tradicionalmente, la pesca es practicada por los matsigenkas durante todo el año, por lo que constituye una importante fuente de proteína en 
su alimentación. De acuerdo con Rosengren (2004), es posible distinguir hasta ocho métodos de pesca con diferentes instrumentos, que emplean los matsigenkas según la época del año y el comportamiento del río. Respecto a la economía moderna, los alumnos matsigenkas señalan que cultivan achiote, café y cacao, destinados a la comercialización. Además, practican la ganadería, algunos participan de la extracción de madera y otros trabajan para empresas petroleras como Pluspetrol o Repsol.

Justamente, los jóvenes prefieren trabajar y son pocos los que apuestan por una educación a nivel superior. Asimismo, las mujeres son más propensas a quedarse en sus comunidades y formar familia a temprana edad. Gracias a un convenio entre la empresa Pluspetrol y la UCSS, los jóvenes matsigenkas son invitados a estudiar cualquiera de las tres carreras que tiene la sede de Atalaya, se les brinda asesoría educativa, seguro de salud entre otros beneficios. De ese modo, se promueve su desarrollo profesional para el bien de ellos mismos y pensando en un futuro aporte para sus comunidades en Cusco.

\subsection{Pueblo yánesha}

Los hablantes se autodenominan "yánesha”, pero el pueblo es conocido también como amoesha o amuesha. La lengua yánesha se habla en la provincia de Chanchamayo y en el departamento de Pasco, donde la lengua es considerada oficial y hay un alfabeto normalizado desde el 2011. Se considera la existencia de dos dialectos y dos grupos sociales. Por ejemplo, en Junín se dice teno "río arriba" y tepo "río abajo"; mientras que en el Alto Palcazu (Pasco) se dice tono y topo, respectivamente (Ministerio de Educación, 2013).

El grado de vulnerabilidad es alto, puesto que la mayoría de los niños no aprenden la lengua en el hogar. Por la violencia terrorista y 
del narcotráfico, muchos tuvieron que migrar hacia las ciudades y eso afectó la continuidad de sus prácticas culturales (Comisión de la Verdad y Reconciliación, 2013). Los alumnos que llegan a la UCSS presentan estas características: algunos están reaprendiendo su cultura para ser docentes bilingües y en la universidad cuentan con el apoyo del profesor Juan López y de alumnos con un mejor dominio de la lengua y su cultura de quienes aprenden en la interacción diaria.

Los yáneshas mantuvieron vínculos con poblaciones andinas desde antes de la llegada de los españoles. Así se evidencia en su mitología y en la existencia de vocablos de origen quechua en el vocabulario de este pueblo (Santos Granero, 1994; Chase Smith, 1999). La colonización en el siglo XVI, no obstante, trajo consigo la disminución de la población nativa debido a enfermedades (Santos Granero, 1994; Mora y Zarzar, 1997). Después de la rebelión de Juan Santos Atahualpa en 1742, se asentaron en el extremo norte del territorio ocupado por este pueblo, en Pozuzo, inmigrantes tiroleses alemanes. Entonces, los yáneshas fueron confinándose a espacios más reducidos.

No obstante, el contacto social y los matrimonios mixtos han generado una población mestiza en la que la lengua y otras manifestaciones culturales van quedando de lado (Santos Granero, 1994; Chase Smith, 1999). Asimismo, la vida de los yánesha fue afectada por el Movimiento Revolucionario Túpac Amaru (MRTA) que comenzó a expandirse hacia la región amazónica, desarrollando sus acciones subversivas, principalmente en las provincias de Oxapampa y Chanchamayo, en donde vivían muchos yáneshas y asháninkas (Comisión de la Verdad y Reconciliación, 2013).

Entre las prácticas económicas tradicionales, se mantiene la de la pesca debido a una mayor disponibilidad de recursos en esta zona. Los yáneshas cuentan con numerosas técnicas de pesca, asociadas al uso del arco y flecha, la tarrafa, los anzuelos metálicos, las trampas, canastas y cercos. Además se 
encuentra el uso de tóxicos naturales como el barbasco (Santos Granero, 2004; Instituto del Bien Común, 2005). En la actualidad, cada vez más las familias yáneshas han adoptado actividades económicas articuladas al mercado regional como el cultivo del café y la crianza de ganado. En los últimos años, la población yánesha en Atalaya ha ido creciendo debido a las oportunidades laborales y académicas, así como las buenas condiciones climatológicas que encuentran ahí. Cada año llegan más alumnos y se caracterizan por el buen nivel académico que demuestran.

\subsection{Pueblo yine}

El pueblo yine fue reconocido por mucho tiempo como "piro". Ocupó desde tiempos prehispánicos la parte alta de la cuenca del río Ucayali y la parte baja del río Urubamba. Por su destreza como navegantes, se sabe que desde la época preinca, los yines se desplazaban largas distancias para intercambiar plumas, cerámicas, pieles y animales vivos por hachas de piedra y metales preciosos con poblaciones diversas (Smith, 2003).

Se sabe que este pueblo tuvo contacto con los españoles a mediados del siglo XVII, con la llegada de misioneros jesuitas y franciscanos a los alrededores del río Tambo y a la parte alta del río Ucayali. La época de auge de la extracción del caucho contribuyó a la disminución de la población de este pueblo. Debido a ello, muchos yines fueron maltratados y esclavizados por los patrones caucheros (ILV, 2006; Smith, 2003).

A mediados del siglo XX, el Instituto Lingüístico de Verano (ILV) llegó a territorio yine. Su finalidad fue la de promover la educación bilingüe intercultural en la zona. Su presencia en comunidades yines habría sido sumamente importante en el mantenimiento de la lengua indígena de este pueblo (Smith, 2003). 
Los yines han participado activamente del proceso de normalización de su lengua. No obstante, en muchas comunidades de Ucayali, ya no se habla el yine como lengua materna y está en proceso de revitalización mediante la escuela. En las comunidades próximas a Sepahua, en cambio, sí se habla. Por su parte, los alumnos yines que llegan a la UCSS son pocos: algunos no conocen su lengua y cultura, otros solo comprenden la lengua y algunos sí son competentes en ella. Frente a esto, la UCSS se ha convertido en un centro de revitalización de las lenguas y culturas originarias de la Amazonía peruana.

\subsection{Pueblo kakinte}

La mayoría de sus hablantes son bilingües. Otros son trilingües y utilizan el asháninka como segundo idioma. Algunos incluso hablan matsigenka. Esta característica les ha permitido hacerse pasar por asháninkas o matsigenkas cuando salen de sus comunidades, las cuales se encuentran alejadas de los principales ríos y centros urbanos. Desde el río Tambo, por ejemplo, desde la comunidad nativa Poyeni, se camina un día y medio para llegar a la zona kakinte. Igualmente desde Cusco, las distancias son largas para acceder a estas comunidades.

Los kakintes han sufrido la violencia de la colonización y del terrorismo al grado de casi desaparecer. Por eso, la población kakinte no es numerosa y existen kakintes en aislamiento voluntario. Ocupar territorios inhóspitos, alejados del centro, ha sido una estrategia para sobrevivir (Comisión de la Verdad y Reconciliación, 2003).

El profesor Esaú Zumaeta, asháninka del río Tambo, comenta que la lengua kakinte se parece mucho al asháninka y por eso es fácil entenderse cuando conversan. En la UCSS, ha habido al menos un alumno kakinte por 
ciclo, pero siempre se han hecho pasar por asháninkas, ya que dominan bien esta lengua y así no tienen que dar mayores explicaciones de quiénes son o dónde han vivido.

\section{Proceso de Construcción de la Identidad Étnica}

Como se ha podido ver en las breves reseñas hechas sobre los pueblos Arawak de donde proceden los alumnos de la UCSS, hay una historia común de contacto inicial entre estos pueblos. Posteriormente, todos sobreviven a las consecuencias nefastas del periodo de colonización. En la actualidad, buscan estrategias para defender su territorio y lograr su libre autodeterminación.

No obstante, cada pueblo es distinto y la primera evidencia de ello es su territorio y su lengua materna. Muchos alumnos desconocían la existencia de otros pueblos y esa relación histórica con sus hermanos Arawak hasta llegar a la universidad. En este nuevo contexto de socialización se da el proceso de construcción de la identidad étnica. De ese modo, la misma universidad fomenta esta construcción al contar con al menos cinco profesores indígenas para que enseñen la gramática de las lenguas originarias y las manifestaciones culturales de cada pueblo.

\subsection{Tradicionalidad frente a modernidad}

La identidad es una característica del individuo que lo diferencia o asemeja a otros individuos formando así un colectivo que se atribuye rasgos significativos. No obstante, una persona puede tener más de una identidad. Las identidades múltiples son un reflejo de la individualidad del sujeto y se contrapone a las "identidades colectivas", que son formaciones grupales. 
Simon (2004) argumenta respecto a las identidades múltiples que estas son una característica de una sociedad moderna donde las distintas ocupaciones e interacciones sociales del individuo lo llevan a desarrollar múltiples identidades que le permitan adecuarse a un mundo cambiante y complejo.

En ese sentido, se puede añadir que muchos jóvenes salen de sus comunidades con una identidad étnica colectiva que es etnocéntrica a tal punto de desconocer o negarse a aceptar la existencia de otros pueblos indígenas. Esta identidad, sin embargo, les permitirá hacer frente al contacto cultural que se da en la universidad. Igualmente, dicha característica les ayudará a ser modelos para quienes están en proceso de recuperación de su cultura.

Ahora bien, la UCSS, sede Atalaya, es un espacio moderno donde la mayoría de conocimientos que se ofrecen al alumno son occidentales. Sin embargo, se exige la recuperación y prácticas culturales para la construcción del perfil de docente bilingüe intercultural que solicita el Estado. En este caso, muchos jóvenes llegan de comunidades tradicionales, no hablan bien el castellano porque dominan mejor su lengua materna. Otros, en cambio, son bilingües coordinados y han logrado un equilibrio cultural gracias al contexto familiar y social en el cual han sido criados y educados. Aunque también hay jóvenes que, al ingresar a la universidad, se avergüenzan de sus orígenes, de su cultura y de su lengua materna o comunitaria.

En ese aspecto, la universidad favorece la interacción entre la tradicionalidad y la modernidad ofreciendo un espacio donde el sujeto puede reconstruir su identidad étnica. No obstante, se debe tener en cuenta que la modernidad se caracterizaría por el predominio del desarrollo de la identidad individual sobre la colectiva. Al respecto, Castells comenta lo siguiente: 
Junto a estas identidades fuertes, comunitarias, aparentemente fundadas en experiencia histórica y tradición cultural, hay también el surgimiento de identidades individuales, autoconstruidas en torno a un proyecto personal, a un principio electivo. Tales identidades individualistas son particularmente importantes en sectores sociales o sociedades en que las identidades comunitarias no se desarrollan, pero en las que los principios abstractos de pertenencia simbólica (tales como ciudadanía, clase social etc.) se debilitan como resultado del vaciamiento de contenido histórico de las instituciones y organizaciones que encarnaban dichos principios (el estadonación, los sindicatos de clase, etc.). (1999, p. 5)

Esto aplicaría a los jóvenes que, tras haber elegido formarse profesionalmente para buscar el desarrollo de sus comunidades, prefieren lograr una meta personal de crecimiento y adecuación a la sociedad nacional dejando de lado la vida comunitaria para instalarse permanentemente en las ciudades. Por tanto, aparece una tensión entre la tradición y la modernidad que afecta la construcción de la identidad, ya que hace que pueda haber no una sino varias, las necesarias para que el o la joven pueda interrelacionarse con éxito en el ámbito social que le toque experimentar.

\section{2 ¿La identidad se construye?}

El sujeto moderno construye su identidad a través de la identificación de sí mismo en su sociedad como un sujeto que forma parte de ella y que a su vez se diferencia de miembros de otras sociedades o grupos culturales 
con los que interactúa. Brubaker y Cooper afirman que la identidad es una condición, la identificación es un proceso.

"Identification" lacks the reifying connotations of "identity". It invites us to specify the agents that do the identifying, and it does not presuppose that such identifying (...) will necessarily result in the internal sameness, the distinctiveness, the bounded groupness that political entrepreneurs may seek to achieve. Identification — of oneself and of others — is intrinsic to social life; "identity" in the strong sense is not. (2000, p. 14)

Giménez (2000) define el término identidad, desde una perspectiva relacional y situacional, como el conjunto de repertorios culturales interiorizados a través de los cuales los actores sociales demarcan simbólicamente sus fronteras y se distinguen de los demás en una situación determinada. Siguiendo a Barth, Cuche explica lo siguiente:

La identidad es una construcción que se elabora en una relación que opone un grupo a los otros con los cuales entra en contacto. Fredrik Barth (1969) fue el pionero de esta concepción de la identidad como manifestación relacional, que permite superar la alternativa objetivismo/subjetivismo. Para Barth, hay que buscar aprehender el fenómeno identitario en el orden de las relaciones entre los grupos sociales. Según él, la identidad es un modo de categorización utilizada por los grupos para organizar sus intercambios. De esta manera, para 
definir la identidad de un grupo, lo que importa no es hacer el inventario del conjunto de los rasgos culturales distintivos, sino encontrar entre estos rasgos los que son empleados por los miembros del grupo para afirmar y mantener una distinción cultural. (2002, pp. 109-110)

La identidad se entiende, entonces, en términos sociales, como un producto social que afecta las relaciones sociales entre los sujetos. El sujeto asume identidades diferentes en diferentes momentos. Estas pueden ser incluso contradictorias, más cuando se es joven, porque las identidades van de la mano con la autoestima y lo que el sujeto quiera proyectar ante otros, cómo se quiera defender de otros o incluso esconderse y pasar desapercibido. El sentimiento de identidad se consolida a través del juego dialéctico entre la semejanza y la diferencia. Cardoso explica que esto debe entenderse "como semejanzas y diferencias de alguien consigo mismo en el transcurso del ○ tiempo, y con los otros en el plano grupal” (2007, p. 91).

\subsection{Arraigo frente a desarraigo}

El mundo en el cual se desenvuelven los jóvenes indígenas actualmente les permite acceder a ámbitos y posibilidades que sus ancestros no tenían. Esto porque las necesidades también han cambiado. En este contexto, los jóvenes tienen la posibilidad de viajar, de migrar. De hecho, el vivir cinco o más años en Atalaya es una muestra de desarraigo, principalmente para quienes viven en zonas muy alejadas y deben quedarse en Atalaya durante sus vacaciones porque simplemente no tienen dinero para viajar. 
La identidad está vinculada al territorio. Muchos incluso poseen un estatus de comuneros y deben volver durante sus vacaciones a sus comunidades para ver sus tierras y administrar sus chacras. Hacerse presente en sus comunidades garantiza su ciudadanía indígena y la pertenencia al lugar. A otros, en cambio, solo los une a la comunidad sus padres. Estos jóvenes quieren viajar, conocer el país y el mundo antes de volver a sus comunidades. Lo cierto es que al egresar, la primera oportunidad laboral que encuentran los jóvenes es trabajar en la provincia de Atalaya. Algunos se van a Junín o Cusco, sin importarles si van a servir a comunidades del pueblo al que pertenecen. Lo importante es simplemente trabajar.

$\mathrm{El}$ arraigo o desarraigo como elemento para construir la identidad depende de la relación que cada joven mantenga con su comunidad y de cómo esta se sostenga a los largo de los años de formación universitaria. Algunas comunidades apoyan económicamente a sus estudiantes, otras en cambio se desentienden por completo. Ante esta situación, la identidad étnica será fundamental para mantener el sentido de pertenencia al grupo étnico.

\subsection{El contexto en la construcción de la identidad}

La construcción de identidad se da en un contexto o situación específicos, cuando el sujeto necesita valerse de este recurso para superar algún inconveniente en su vida social o simplemente distinguirse de sus pares. Cuando los jóvenes de distintos pueblos se encuentran en el espacio universitario, se produce una dialéctica que conlleva a los jóvenes a preguntarse por las diferencias y semejanzas culturales que tienen con sus compañeros. 


\section{LILIANA FERNÁNDEZ FABIÁN}

La construcción de las identidades se puede entender dentro de un conjunto de condiciones y momentos. El momento de producción de la identidad es también el momento de reproducción de los contextos donde se escenifica (expresa) dicha identidad. Los contextos se relacionan con la vida cotidiana de los sujetos, de ahí que en los movimientos indígenas, la comunidad, el territorio histórico y la organización sean un producto de las relaciones sociales y los intereses colectivos, al mismo tiempo que el escenario en que se produce y cobra sentido aquello que los sujetos entienden como su identidad social. (Bello, 2004, p. 31)

Se entiende que cada grupo selecciona aquello que considera propio o ajeno, de acuerdo al momento, a las condiciones políticas y económicas particulares de su proceso histórico. La cultura es una fuente de recursos que emplea el individuo para construir una serie de identidades que le permita sostenerse en un determinado grupo social. Eventos como la semana de la Educación Intercultural bilingüe, el aniversario de Atalaya o la visita de alguna autoridad son escenarios propicios para construir la identidad étnica.

Parte de la evaluación para ingresar a la UCSS, sede Atalaya, es una entrevista en lengua indígena, en la cual el postulante demuestra el conocimiento que tiene de su lengua y cultura. Además, la vestimenta forma parte de la evaluación. Estas condiciones exigen que los alumnos se embellezcan con sus mejores atuendos y ornamentas. Es un contexto que exige hacer visible la identidad de manera material. En cambio, otros contextos, como la vida comunal, exigirán del individuo una representación simbólica que se hace patente en sus creencias y costumbres, en la moral acorde a las reglas de su pueblo. 


\subsubsection{La universidad}

La UCSS constituye el espacio atalaíno en el cual los estudiantes se encuentran así como se reconocen y diferencian. Lo primero que llama la atención de todos es la vestimenta indígena tradicional. Tantos diseños nunca antes vistos, tantos colores y ornamentas, la pintura fácil, los tocados, los bolsos tan diferentes les hacen comprender su pertenencia a pueblos distintos, pero luego observan algún toque similar gracias al cual entienden que son Arawak.

Aunque la universidad les exige usar la vestimenta tradicional durante las clases y en las ceremonias oficiales, muchos recién están reconociendo qué los diferencian de los demás. Esto sucede durante las clases de las distintas materias que llevan y en los minutos de receso donde interactúan entre ellos, averiguan sus procedencias, reconocen léxico común, encuentran familia, vecinos y se organizan por etnias.

La construcción de la identidad se hace en el interior de los marcos sociales que determinan la posición de los agentes y por lo tanto orientan sus representaciones y sus elecciones. Por otra parte, la construcción identitaria no es una ilusión, pues está dotada de una eficacia social, produce efectos sociales reales. (Cuche, 2002, p. 109)

Los sujetos ponen en práctica estrategias específicas en el proceso de construcción de su identidad o identidades en un contexto que incluye la historicidad y las prácticas culturales, además de lo social, es decir, las relaciones sociales y relaciones de poder. Por eso, los jóvenes necesitan aprender todo acerca de su cultura: historia y competencia total en la lengua 


\section{LILIANA FERNÁNDEZ FABIÁN}

para ser de uno u otro pueblo. La aceptación del individuo no siempre está garantizada por el hecho de que alguno de sus progenitores o abuelos sea indígena. Entonces, la sola voluntad del joven lo sitúa en un contexto favorable para construir su identidad. Respecto a las estrategias que empleará para cumplir sus objetivos, es interesante lo que señala Cuche:

(...) el concepto de estrategia puede explicar las variaciones identitarias, lo que se podría denominar los desplazamientos de la identidad. Muestra la relatividad de los fenómenos de identificación. La identidad se construye, se desconstruye y se reconstruye según las situaciones. Está en un continuo movimiento; cada cambio social la lleva a reformularse de una manera diferente. (2002, p. 119)

Debido a contextos como este, Simon (2004) señala que existen procesos psicosociales que operan a favor de las funciones de la identidad. Ello se da en el sentido que ayudan a las personas a alcanzar, mantener o presentar una identidad respetada: "processes of self-enhancement operate in the service of the respect function because they help people to achieve, maintain or present a respected identity. With regard to collective identity, prejudice and discrimination processes are particularly relevant here" (Simon, 2004, p. 68).

Pero, además, no hay que olvidar que el rol del estatus social juega un papel muy importante en la configuración de la identidad y en la forma en que se llevan a cabo los procesos ya descritos: "The experience of being collectively stereotyped may lead members of low-status minorities to recognize, accept and develop similarities with other ingroup members which then become part and parcel of their self-stereotypes and thus 
provide a basis for collective identity” (Simon, 2004, p. 107). Así, cada grupo empieza a unificar preconceptos respecto a sus pares: "Los asháninkas son más guerreros que nosotros", "Los yáneshas somos líderes y sabemos organizarnos", "Los matsigenkas son muy nobles", "Los yines son buenos comerciantes", etc.

\subsubsection{Los cursos de lengua y artesanía}

Las clases de Lengua indígena son el espacio perfecto para que los jóvenes se reencuentren con su cultura y su lengua. Se exige que los alumnos asistan vestidos con su indumentaria tradicional a estas clases que se dan en lengua originaria con traducción al español cuando hay alumnos que no comprenden la lengua. Entonces, se dan cuenta de que su lengua materna es tan importante como el castellano, que su lengua también es un sistema y es gramática: así como el castellano tienen verbo y sustantivo, sus lenguas también cuentan con estas categorías. Incluso, comprenden que sus lenguas tienen posposiciones y el castellano no.

Los alumnos nomatsigenga y kakinte llevan clases con los asháninkas o ashéninkas y tienen que establecer comparaciones de la gramática que aprenden con su lengua. Cabe agregar que no todos se muestran conformes, puesto que han postulado a la UCSS, sede Atalaya, para aprender más sobre su lengua y cultura, pero eso no se está dando. En este sentido, la participación del profesor de Lengua indígena es fundamental como sabio y guía en el proceso de formación académica de los alumnos.

Hasta el año 2015, Lengua y Artesanía eran dos cursos distintos: el primero tenía seis horas pedagógicas a la semana y el segundo era un taller de una hora semanal. En la actualidad ambos cursos se han fusionado y algunos alumnos no están conformes porque ya no tienen tiempo para hacer 
artesanía. Anteriormente, muchos aprendieron en estas clases sobre antiguas herramientas e instrumentos de caza como el arco y la flecha. En ese caso, conocer manifestaciones culturales que ellos ya no han podido practicar es importante en el proceso de construcción de su identidad.

Las clases de Lengua indígena no solo tienen un enfoque gramatical. En estos cursos, los docentes recapitulan sus historias. Apelan a una historia o relato que se ha transmitido oralmente, reconocen sus espacios ancestrales, el proceso de colonización y aprenden sobre la diversidad cultural al interior de cada pueblo Arawak.

\subsection{Fortalecimiento cultural y revitalización lingüística}

La identidad colectiva es resultado de la configuración de la identidad o identidades individuales. En el caso de los alumnos de la UCSS, sede Atalaya, a partir del contacto sociocultural entre jóvenes con distintos tipos de experiencias en sus comunidades nativas Arawak, se da el fortalecimiento de la identidad colectiva mediante el conocimiento de su historia y sus patrones culturales tradicionales. La unidad del grupo hace que los integrantes más débiles se fortalezcan y con ello la identidad étnica se reafirme.

La identidad colectiva se alimenta de un conjunto de identidades individuales y estas, a su vez, derivan de la identidad colectiva. Se trata de un proceso constante que solo se evidencia cuando el sujeto es cuestionado respecto a esto por las circunstancias socioculturales a las que se expone. Por esa razón, muchos jóvenes se encuentran en el proceso de fortalecer esta identidad cultural gracias a la interacción diaria con sus pares:

(...) identity appears to be a matter of choice, although the underlying belief seems to be that just as the collectivity has an 
authentic identity that will emerge in time, so the individual has a necessary identity with a particular cultural collectivity, even if it remains to be discovered, perhaps after a period of denial. (Kuper, 2000, p. 238)

Asimismo, es interesante el proceso de revitalización lingüística que se da con las clases de lengua y apertura de espacios dentro de la universidad. Gracias a todo esto, los alumnos son libres para emplear sus lenguas originarias. Así, muchos alumnos, al egresar, ya han adquirido la lengua indígena como segunda lengua y pueden ir a las comunidades a enseñar.

De igual modo, los docentes indígenas cumplen un rol fundamental en este proceso de revitalización que se da de forma explícita durante tres años. Sus conocimientos sobre la historia de sus pueblos, geografía, cosmovisión y lengua, los convierten en guías del proceso de formación de sus alumnos. Más aún, ven en ellos a consejeros y los frecuentan hasta el último ańo de carrera para corroborar alguna traducción o pedir que los escuchen y orienten.

\section{Conclusiones}

La construcción social de la identidad de los distintos pueblos Arawak en la UCSS, sede Atalaya, se lleva a cabo en un contexto marcado por la interculturalidad como un proceso permanente de reconocimiento y aceptación colectiva e individual. Muchos alumnos se reencuentran consigo mismos en la universidad, se aceptan tal como son. Asimismo, aprenden a enorgullecerse de sus culturas, sus padres y demás paisanos.

Como institución dominante, la universidad permite el diálogo intercultural entre los pueblos y exige la promoción cultural a través de la 
recuperación de tradiciones, la revitalización de las lenguas y la competencia en lengua indígena de sus alumnos. Así, por ejemplo, es la única universidad que ha respetado al pueblo ashéninka y lo ha reconocido como tal con sus propias características culturales y lengua distinta al del asháninka. Para ese caso, la lengua ashéninka se enseña con su propio alfabeto, distinto al oficializado en el 2008. De esa forma, los alumnos se sienten respetados y reconocidos porque tienen un propio alfabeto. Participan como ashéninkas de las actividades extracurriculares y hacen gala de sus trajes típicos y pinturas faciales que claramente los hacen distintos de los asháninkas.

Gracias a esta formación, los alumnos obtienen las herramientas necesarias para defenderse ante la sociedad nacional, ya que son capaces de construir una identidad fortalecida gracias al conocimiento de la realidad nacional, regional y local que adquieren en este centro de estudios. Al mejorar su autoestima, y sentirse parte de una sociedad rica en diversidad cultural y lingüística, logran posicionarse mejor en su entorno y enseñar en ámbitos interculturales bilingües sobre la base del respeto a la diferencia sin negar la condición del otro.

El mundo actual supone interacciones de un nuevo orden e intensidad, distintas al pasado en el cual las transacciones culturales entre grupos sociales se limitaban geográfica y ecológicamente. En la actualidad, el modo de vida tradicional ha pasado a segundo plano para muchos jóvenes, ya que el ámbito social exige que presten atención a otras obligaciones, principalmente, el estudiar para servir a su comunidad como profesional o líder político. Frente a ello, los jóvenes que egresan de la UCSS, sede Atalaya, se reconocen como modelos de modernidad para los niños y jóvenes que conocerán en sus caminos. Por esa razón, llevan orgullosos el título de docentes bilingües interculturales. 


\section{Referencias}

Asociación Interétnica de Desarrollo de la Selva Peruana, Programa de Formación de Maestros Bilingües de la Amazonía Peruana, Fundación Telefónica. (2000). El ojo verde. Cosmovisiones amazónicas. Lima, Perú: Autor.

Barclay, F. (2006). La ocupación de la Amazonía central: un proceso continuo de negación de derechos y expropiación territorial. En M. Benavides (Ed.), Atlas de comunidades nativas de la selva central (pp. 26-27). Lima, Perú: Instituto del Bien Común.

Barth, F. (Ed.). (1969). Ethnic groups and boundaries. The social organization of culture difference. Boston, Estados Unidos: Little, Brown and Company.

Bello, A. (2004). Etnicidad y ciudadanía en América Latina. La acción colectiva de los pueblos indigenas. Santiago de Chile, Chile: CEPAL, GTZ.

Brubaker, R., \& Cooper, F. (2000). Beyond "identity". Theory and Society, $29,1-47$.

Cardoso, R. (2007). Etnicidad y estructura social. México D. F., México: CIESAS, Universidad Autónoma Metropolitana, Universidad Iberoamericana.

Castells, M. (1999). Globalización, identidad y Estado en América Latina. Santiago de Chile, Chile: Programa de Naciones Unidas para el Desarrollo. Recuperado de http://www.desarrollohumano.cl/ otraspub/Pub01/Idyest.pdf 


\section{LILIANA FERNÁNDEZ FABIÁN}

Chase Smith, R. (1999, mayo). Caciques chinchaycochas, funcionarios incas y sacerdotes amueshas: los caminos antiguos de Chinchaycocha hacia la selva central. Ponencia presentada en la conferencia La cultura de Pasco. Universidad Daniel Alcides Carrión, Cerro de Pasco.

Comisión de la Verdad y Reconciliación. (2003). Los pueblos indígenas y el caso de los asháninkas. Informe Final de la Comisión de la Verdady Reconciliación (T. V, pp. 241-277). Lima, Perú: Autor.

Cuche, D. (2002). Cultura e identidad. En Autor, La noción de cultura en las ciencias sociales (pp. 105-122). Buenos Aires, Argentina: Nueva Visión.

Espinosa, O. (1993). Las rondas asháninka y la violencia política en la selva central. América Indígena, 53(4), 79-101.

Giménez, G. (2000). Materiales para una teoría de las identidades sociales. En J. M. Valenzuela Arce (Coord.), Decadencia o auge de las identidades. Cultura nacional, identidad cultural y modernización (pp. 45-78). México D. F., México: El Colegio de la Frontera de México y $\mathrm{P} \& \mathrm{~V}$ coeditores.

Hvalkof, S., \& Veber, H. (2005). Los ashéninka del Gran Pajonal. En Santos Granero, F. y Barclay, F. (Eds.), Guía etnográfica de la Alta Amazonia. Campa Ribereños/Ashéninka (Vol V., pp. 75-279). Lima, Perú: Instituto Francés de Estudios Andinos.

Instituto del Bien Común. (2005). Los yanesha y la pesca. Lima, Perú: Autor. Instituto Lingüístico de Verano. (2006). Pueblos del Perú. Lima, Perú: Autor. (Reimpreso de People of Peru, 1999). 
Instituto Nacional de Estadística e Informática. (2007). II Censo de Comunidades Indígenas de la Amazonía Peruana 2007. Resultados definitivos. Lima, Perú: Instituto Nacional de Estadística e Informática.

Mayor, P., \& Bodner, R. (2009). Pueblos indigenas de la Amazonía peruana. Iquitos, Perú: Centro de Estudios Teológicos de la Amazonía.

Ministerio de Educación. (2013). Documento nacional de lenguas originarias del Perú. Lima, Perú: Autor.

Mora, C., \& Zarzar, A. (1997). Información sobre familias lingüísticas y etnias en la Amazonía peruana. En A. Brack (Ed.), Amazonía peruana, comunidades indigenas, conocimientos y tierras tituladas: Atlas y base de datos (pp. 29-141). Lima, Perú: Global Environment Facility, Programa de las Naciones Unidas para el Desarrollo, Oficina de las Naciones Unidas de Servicios para Proyectos.

Rosengren, D. (2004). Los matsigenka. En F. Santos Granero \& F. Barclay (Eds.), Guía etnográfica de la Alta Amazonía. (Vol. IV, pp.1-158). Lima, Perú: Instituto Francés de Estudios Andinos.

Santos Granero, F. (2004). Los yanesha. En F. Santos Granero \& F. Barclay (Eds.), Guía etnográfica de la Alta Amazonía (Vol. IV, pp. 159-360). Lima, Perú: Instituto Francés de Estudios Andinos.

Santos Granero, F. (1994). El poder del amor. Poder, conocimiento y moralidad entre los amuesha de la selva central del Perú. Quito, Ecuador: Abya Yala. 
Shepard, G., \& Izquierdo, C. (2003). Los matsiguenka de Madre de Dios y del Parque Nacional del Manu. En B. Huertas \& A. García (Eds.), Los pueblos indigenas de Madre de Dios (pp. 111-126). Lima, Perú: Federación Nativa del Río Madre de Dios y Afluentes, International Work Group for Indigenous Affairs.

Simon, B. (2004). Identity in modern society. A social psychological perspective. Oxford, United Kingdom: Blackwell Publishing.

Smith, A. (2003). Del ser piro y el ser yine. Apuntes sobre la identidad, historia y territorialidad del pueblo indígena yine. En B. Huertas \& A. García (Eds), Los pueblos indigenas de Madre de Dios (pp. 127-143). Lima, Perú: Federación Nativa del Río Madre de Dios y Afluentes, International Work Group for Indigenous Affairs. 